\title{
Use of the complete basis set limit for computing highly accurate $a b$ initio dipole moments
}

Cite as: J. Chem. Phys. 152, 024105 (2020); https://doi.org/10.1063/1.5135931

Submitted: 08 November 2019 . Accepted: 19 December 2019 . Published Online: 08 January 2020

Eamon K. Conway (D), Iouli E. Gordon (D), Oleg L. Polyansky, and Jonathan Tennyson (D)

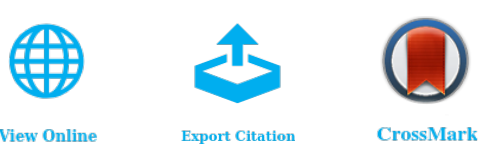

\section{ARTICLES YOU MAY BE INTERESTED IN}

Adventures in DFT by a wavefunction theorist

The Journal of Chemical Physics 151, 160901 (2019); https://doi.org/10.1063/1.5116338

Dissipation and dynamics in ultrafast intersystem crossings

The Journal of Chemical Physics 152, 024104 (2020); https://doi.org/10.1063/1.5125005

Fantasy versus reality in fragment-based quantum chemistry

The Journal of Chemical Physics 151, 170901 (2019); https://doi.org/10.1063/1.5126216

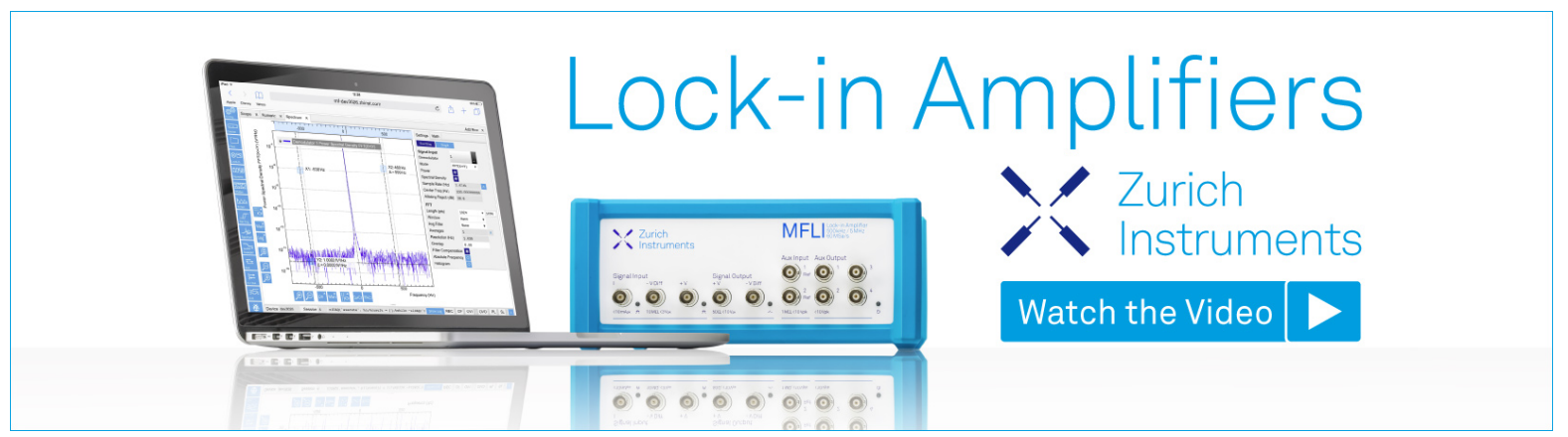




\title{
Use of the complete basis set limit for computing highly accurate $a b$ initio dipole moments
}

\author{
Cite as: J. Chem. Phys. 152, 024105 (2020); doi: 10.1063/1.5135931 \\ Submitted: 8 November 2019 • Accepted: 19 December 2019 • \\ Published Online: 8 January 2020
}

\author{
Eamon K. Conway, ${ }^{1,2, a)}$ (D) Iouli E. Gordon, ${ }^{1}$ (D) Oleg L. Polyansky, ${ }^{2}$ and Jonathan Tennyson
}

\begin{abstract}
AFFILIATIONS
${ }^{1}$ Harvard \& Smithsonian | Center for Astrophysics, Atomic and Molecular Physics Division, Cambridge, Massachusetts O2138, USA ${ }^{2}$ Department of Physics and Astronomy, University College London, Gower Street, London WC1E 6BT, United Kingdom
\end{abstract}

a) Author to whom correspondence should be addressed: eamon.conway@cfa.harvard.edu

\begin{abstract}
Calculating dipole moments with high-order basis sets is generally only possible for the light molecules, such as water. A simple, yet highly effective strategy of obtaining high-order dipoles with small, computationally less expensive basis sets is described. Using the finite field method for computing dipoles, energies calculated with small basis sets can be extrapolated to produce dipoles that are comparable to those obtained in high order calculations. The method reduces computational resources by approximately $50 \%$ (allowing the calculation of reliable dipole moments for larger molecules) and simultaneously improves the agreement with experimentally measured infrared transition intensities. For atmospherically important molecules, which are typically too large to consider the use of large basis sets, this procedure will provide the necessary means of improving calculated spectral intensities by several percent.
\end{abstract}

Published under license by AIP Publishing. https://doi.org/10.1063/1.5135931

\section{INTRODUCTION}

The ability of atmospheric science missions to accurately detect trace amounts of molecules in our atmosphere is placing significant demands on spectroscopy, ${ }^{1-5}$ both theoretical and experimental. For instance, spectrometers on board of satellites including GOSAT $^{6}$ and OCO- $2^{7}$ now aim to detect carbon dioxide in the terrestrial atmosphere to an accuracy better than $0.3 \%$. Achieving this precision requires all line parameters to be known to very high accuracy. Atmospheric missions rely on the spectroscopic data in the high-resolution transmission molecular absorption (HITRAN) database, ${ }^{8}$ which typically combines experimental and theoretical data in order to achieve both completeness and accuracy of reference line lists. Every four years, the HITRAN parameters are being updated and extended to incorporate state of the art data.

Modern experimental methods, especially those that employ frequency combs (see, for instance, Refs. 9-11), can measure line positions with errors on the order of $10^{-5} \mathrm{~cm}^{-1}$, if not smaller, which is several orders of magnitude better than the accuracy that can be achieved by calculations using the best semiempirical potential energy surfaces available. ${ }^{12-14}$ For example, for water vapor, the most accurate potential energy surface available ${ }^{14}$ predicts energy levels below $15000 \mathrm{~cm}^{-1}$ with a standard deviation $\sigma=0.011 \mathrm{~cm}^{-1}$. These high-accuracy semiempirical potentials are achieved via a process known as refinement. ${ }^{15}$ When experimental data are available, the parameters used in fitting the underlying electronic structure calculations are adjusted to improve the agreement between theory and the observed data. This procedure, when done correctly, allows energy levels to be predicted to less than $0.03 \mathrm{~cm}^{-1}$; hence, the accuracy is no longer proportional to the level of theory considered for the electronic structure calculations. ${ }^{13,14,16,17}$ However, when refinement is not possible, then the level of theory becomes significantly more important. Extrapolating calculated energies to the complete basis set (CBS) is one such method that has been used with great success. Second order corrections such as relativistic, adiabatic, nonadiabatic, quantum electrodynamics, and spin orbit coupling can also become important. ${ }^{1}$

Cavity ring down spectroscopy can measure those transitions that have very weak intensities with high accuracy. ${ }^{22}$ For wellstudied molecules including $\mathrm{H}_{2} \mathrm{O}$ and $\mathrm{CO}_{2}$, transition intensities obtained from $a b$ initio calculations and experiments are generally in excellent agreement with deviation on the sub-one-percent scale for many bands.

For a given method, the basis set chosen for the electronic structure calculations largely determines the accuracy of 
an $a b$ initio dipole calculation. The Dunning ${ }^{26-28}$ aug-cc-pCV6Z basis set is the largest conventional basis set yet considered for the calculation of a global dipole surface $^{29,30}$ for a triatomic system, in this case water. Each of these dipole calculations required almost two days worth of central processing unit (CPU) time to compute; ${ }^{29,30}$ hence, for molecules with more electrons, such as $\mathrm{CO}_{2}$, use of the aug-cc-pCV6Z basis is currently unfeasible.

Even with the extensive computer resources used in the creation of accurate dipole moment surfaces (DMS's) for water, over 100 years of CPU time in the case the CKAPTEN DMS, ${ }^{29}$ there still remains issues with predicted infrared transition intensities. For example, the $v_{1}$ fundamental has been shown to be very sensitive to the choice of $a b$ initio calculations ${ }^{25,29,31,32}$ and improvements are needed. Computing dipoles with a larger basis set is one solution, although not feasible on a global scale. Similar problems persist for other atmospherically important molecules such as $\mathrm{CO}_{2}{ }^{33}$ and ozone. $^{34,35}$

This work aims to explore the use of complete basis set (CBS) extrapolation in the computation of high-accuracy $a b$ initio spectral intensities both to give increased accuracy and to reduce the computational cost.

\section{METHOD}

\section{A. Calculations}

We have computed over 16000 aug-cc-pCV6Z ${ }^{26-28}$ finitefield dipoles for water using the electronic structure program MOLPRO. ${ }^{36}$ With these, we created a DMS for water vapor, named CKAPTEN. ${ }^{29}$ Spectra computed with this CKAPTEN surface have been shown to produce excellent transition intensities when compared to both experiment and observation. ${ }^{37}$ From these 16000 configurations, we create a smaller subgrid with 2540 data points. The points were chosen such that there is sufficient coverage of both stretching and bending coordinates, which would provide accurate transition intensities up to approximately $15000 \mathrm{~cm}^{-1}$. For calculating reliable spectra in the ultraviolet, it is necessary to limit the use of fitting parameters as done in CKAPTEN, which reduces the possibility of artificial oscillations occurring in highly energetic regions of the dipole surface. In this work, we are not interested in such energetic transitions and are not required to use a few parameter fit nor to use 16000 points to better constrain the fit. The stretching coordinates in this subgrid are in the range of $1.4 \mathrm{a}_{0}-4 \mathrm{a}_{0}$, while the angular selection lies between $30^{\circ}$ and $178^{\circ}$. With respect to our equilibrium configuration of $1.8141 \mathrm{a}_{\mathrm{o}}$ and $104.52^{\circ}$, our dataset includes dipoles with energies up to $42481 \mathrm{~cm}^{-1}$. The original 16000 finite-field dipoles were computed at the multireference configuration interaction (MRCI) level with the aug-cc-pCV6Z basis set, a Davidson correction (+Q), and Douglass-Kroll-Hess-Hamiltonian to order two (DKH2) and with an electric field strength of $5 \times 10^{-5}$ a.u. We calculate finite-field dipoles on our subgrid of 2540 points that use smaller basis sets, notably the aug-cc-pCV(Q,5)Z sets, while still using the same formalism employed for the aug-cc-pCV6Z dipoles. Calculations were all performed on Legion, Grace, and Myriad systems at the University College London High Performance Computing Facilities, and CPU times given below are for these computers.

\section{B. Extrapolation technique}

We configure our water molecule such that the $\mathrm{z}$-axis bisects the angle $\mathrm{HOH}$, with the $\mathrm{x}$-axis in the $\mathrm{HOH}$ plane and perpendicular to $z$. In the finite field approach, we apply a small electric field, $\lambda$, in each of the directions: $+\hat{x},-\hat{x},+\hat{z}$, and $-\hat{z}$. The energy of the molecule in each respective field will be termed $E_{p x}, E_{n x}, E_{p z}$, and $E_{n z} \cdot p$ and $n$ signify positively and negatively directed fields, respectively, while $x$ and $z$ are the components. Each dipole component is calculated as

$$
\mu_{x(z)}=\frac{\left(E_{p x(p z)}-E_{n x(n z)}\right)}{2 \lambda} .
$$

We extrapolate the individual energies $E_{p x}, E_{n x}, E_{p z}$, and $E_{n z}$ with a standard expression $^{38}$

$$
E_{x}=E_{c b s}+b e^{-x},
$$

where $E_{c b s}$ and $b$ are fitted parameters, while $x$ represents the level of theory. This will provide us with $E_{p x}^{c b s}, E_{n x}^{c b s}, E_{p z}^{c b s}$, and $E_{n z}^{c b s}$. This formula (2) has successfully been used in the past to extrapolate $a b$ initio energies for potential energy surfaces. ${ }^{13,21,39}$ Instead of one single energy extrapolation to do, we have five: the zero-field calculation and one for each of our four dipole components within an electric field.

We are interested in applying this technique to produce extrapolated dipoles, $\mu_{x(z)}^{c b s_{Q 5}}$ and $\mu_{x(z)}^{c b s_{56}}$. We expect the $\mu_{x(z)}^{c b s_{Q 5}}$ dipoles to behave as the aug-cc-pCV6Z dipoles and the $\mu_{x(z)}^{c b s_{56}}$ dipoles to behave as aug-cc-pCV7Z dipoles.

To create $\mu_{x(z)}^{c b s_{Q 5}}$ dipoles, we require $E_{p x}^{c b s_{Q 5}}, E_{n x}^{c b s_{Q 5}}, E_{p z}^{c b s_{Q 5}}$, and $E_{n z}^{c b s_{Q 5}}$, which represent the individually extrapolated energies. Likewise, to calculate $\mu_{x(z)}^{c b s_{56}}$, we need $E_{p x}^{c b s_{56}}, E_{n x}^{c b s_{56}}, E_{p z}^{c b s_{56}}$, and $E_{n z}^{c b s_{56}}$. Combining these values, we can now calculate our extrapolated dipoles,

$$
\mu_{x(z)}^{c b s}=\frac{\left(E_{p x(p z)}^{c b s}-E_{n x(n z)}^{c b s}\right)}{2 \lambda} .
$$

In total, we possess five sets of dipoles, one for each of the augcc-pCV $(\mathrm{Q}, 5,6) \mathrm{Z}$ basis sets and then two sets of extrapolated sets, $\mathrm{CBS}^{\mathrm{Q} 5}$ and $\mathrm{CBS}^{56}$.

\section{Fitting}

We fit each dipole set to a similar functional form used in the creation of CKAPTEN, ${ }^{29}$

$$
\begin{gathered}
\mu_{z}\left(r_{1}, r_{2}, \theta\right)=(\pi-\theta) \sum_{i, j, k} C_{i j k}^{(z)} \zeta_{1}^{i} \zeta_{2}^{j} \zeta_{3}^{k}, \\
\mu_{x}\left(r_{1}, r_{2}, \theta\right)=\sum_{i, j, k} C_{i j k}^{(x)} \zeta_{1}^{i} \zeta_{2}^{j} \zeta_{3}^{k},
\end{gathered}
$$

where $\zeta_{1}=\frac{\left(r_{1}+r_{2}\right)}{2}-r_{e}, \zeta_{2}=\left(r_{2}-r_{1}\right)$, and $\zeta_{3}=\theta / \theta_{e}$. To physically model the dipole surface correctly, we have several conditions that must be adhered to the following requirements:

(i) $\mu_{z}\left(r_{1}, r_{2}, \theta=\pi\right)=0$,

(ii) $\mu_{z}\left(r_{1}, r_{2}, \theta\right)=\mu_{z}\left(r_{2}, r_{1}, \theta\right)$, and

(iii) $\mu_{x}\left(r_{1}, r_{2}, \theta\right)=-\mu_{x}\left(r_{2}, r_{1}, \theta\right)$. 
TABLE I. The weighted root-mean-square (rms) deviation of each fitted dipole component for each surface (see text for details).

\begin{tabular}{lcc}
\hline \hline & $\mu_{z}$ (a.u.) & $\mu_{x}$ (a.u.) \\
\hline Q-zeta & $7.29 \times 10^{-5}$ & $1.10 \times 10^{-4}$ \\
5-zeta & $5.55 \times 10^{-5}$ & $9.95 \times 10^{-5}$ \\
CBS(45) & $8.59 \times 10^{-5}$ & $1.20 \times 10^{-4}$ \\
6-zeta & $5.35 \times 10^{-5}$ & $9.96 \times 10^{-5}$ \\
CBS(56) & $7.77 \times 10^{-5}$ & $1.10 \times 10^{-4}$ \\
\hline \hline
\end{tabular}

These requirements mean that only even (odd) powers of $j$ are used in the expression $\zeta^{j}$ for the $\hat{z}(\hat{x})$ components.

The dipoles in each set are weighted ${ }^{31,32}\left(w_{i}\right)$ as a function of their energy,

$$
\begin{gathered}
s_{i}=\tanh \left(-0.006\left(E_{i}-30000\right)+1.002002002\right) / 2.002002002 \\
w_{i}=25000\left(s_{i}\right) / \max \left(E_{i}, 25000\right) .
\end{gathered}
$$

To facilitate an equal comparison between the different levels of theory, each dipole set must be fit to the same functional form, requiring the same number of parameters in each fit. We fit the $\hat{z}$ components with 228 parameters and the $\hat{x}$ with 163 parameters.

Table I shows the average root-mean-square deviation of the dipole fitting procedure for each of the individual surfaces. For the parallel $\hat{z}$ component, each surface is fit to a root-mean-square (rms) under $10^{-4}$ a.u., while for the perpendicular $\hat{x}$ component, each surface possesses an rms of approximately $1.1 \times 10^{-4}$ a.u. The perpendicular component is often the most difficult to fit and carries a larger rms than the respective parallel component of the dipole $^{29,30}$

To compare the surfaces, we need to assess the resulting transition intensities. To do this, we require wavefunctions, which we calculate from the potential energy surface of Mizus et al. ${ }^{14}$ This PES, called PES15K, is valid for energies that fall below $15000 \mathrm{~cm}^{-1}$, which is sufficient for our study. For states falling below this threshold, PES15K predicts energies to a rms of only $0.011 \mathrm{~cm}^{-1}$. Using the DVR3D ${ }^{42}$ suite of programs, we calculate spectra for each of the five dipole surfaces with $J_{\max }=6, v_{\max }=15000 \mathrm{~cm}^{-1}, S_{\text {if }} \geq 10^{-30} \mathrm{~cm}$ molecule ${ }^{-1}$, and $T=296 \mathrm{~K}$.

\section{RESULTS}

Table II contains energies $E_{p x}, E_{n x}, E_{p z}$, and $E_{n z}$ with the corresponding dipoles $\mu_{x}$ and $\mu_{z}$ obtained using basis sets aug-cc$\operatorname{pCV}(\mathrm{X}=\mathrm{Q}, 5,6) \mathrm{Z}$ on one molecular geometry: $R_{1}=1.7 \mathrm{a}_{\mathrm{o}}, R_{2}=1.98$ $\mathrm{a}_{\mathrm{o}}$, and $\theta=163^{\circ}$. Attempts to extrapolate dipoles $\mu_{z, x}$ for the aug-cc-pCV $(\mathrm{X}=\mathrm{Q}, 5) \mathrm{Z}$ basis sets with formula $\mu_{x, z}=\mu_{x, z}^{c b s}+b e^{-x}$ result in failure. The best fit to these dipoles is a linear function with $\chi^{2}$ on the order of $10^{-12}$, which is incorrect: energies can be extrapolated to their complete basis set limit and dipoles growing linearly with growth in basis set size cannot be extrapolated.

Many extrapolation schemes exist, and it is often suggested to extrapolate the Hartree-Fock energy component separately from the correlation contribution as they can converge at different rates. ${ }^{44}$ However, for the data in Table II, the rate of convergence of their combined energy is described best by an exponential curve. Extrapolating the components independently with different schemes should not provide drastically different results on the dipoles; hence, we estimate the fit uncertainty on the transition intensities to be on the subpercent scale.

For the energy extrapolations, the fitted $b$ variables are found to be equal for extrapolating $E_{p z}$ and $E_{n x}$ energies and also for $E_{p x}$ and $E_{n z}$. The $\mu_{z, x}$ dipoles for QZ, $5 Z$, and $6 Z$ shown in Table II were computed within the program MOLPRO using energies that were converged to the tenth significant figure; hence, these dipoles are accurate to the sixth significant figure (dividing by $2 \lambda$ is equivalent to multiplying by 10000 ). The individual energies, $E_{p x}, E_{n x}, E_{p z}$ and $E_{n z}$, were written out to only eight significant figures; hence, the extrapolated dipoles that use these energies are instead accurate to the fourth significant figure.

Table III shows eight selected molecular configurations and presents the dipole values calculated for each of the five theoretical methods with the average CPU time required per configuration. For the extrapolated surfaces, we combine the CPU time for each of the individual calculations required for the extrapolation to provide a total time. The $\mathrm{CBS}^{\mathrm{Q} 5}$ dipoles deviate by less than $10^{-4}$ a.u. from the $\mathrm{CBS}^{56}$, which was not expected, yet these $\mathrm{CBS}^{\mathrm{Q} 5}$ dipoles only

TABLE II. Calculated dipole moment and four energies $E_{p x}, E_{n x}, E_{p z}$, and $E_{n z}$ with the molecular configuration consisting of $R_{1}=1.7 \mathrm{a}_{0}, R_{2}=1.98 \mathrm{a}_{0}$, and $\theta=163^{\circ}$ computed with basis sets aug-cc-pCV $(\mathrm{X}=\mathrm{Q}, 5,6) Z$. The calculated dipoles carry more precision than the energies shown here. $Q Z$ and $5 Z$ energies are extrapolated with $E=E_{c b s}+b e^{-x}$, and the respective $\mathrm{CBS}^{\mathrm{Q5}}$ dipoles are calculated.

\begin{tabular}{lccccc}
\hline \hline & $\mathrm{QZ}$ & $5 Z$ & $6 Z$ & $\mathrm{CBS}$ & $b$ \\
\hline$E_{p z}$ & -76.41695707 & -76.42504517 & -76.42813805 & -76.42975226 & 0.69859347 \\
$E_{n z}$ & -76.41697909 & -76.42506722 & -76.42816013 & -76.42977432 & 0.69859606 \\
\hline$\mu_{z}$ & 0.220221 & 0.220487 & 0.220746 & 0.220675 & $\ldots$ \\
\hline$E_{p x}$ & -76.41697514 & -76.42506327 & -76.42815617 & -76.42977037 & 0.69859606 \\
$E_{n x}$ & -76.41696102 & -76.42504912 & -76.42814201 & -76.42975620 & 0.69859347 \\
\hline$\mu_{x}$ & -0.141258 & -0.141482 & -0.141600 & -0.141675 & $\ldots$ \\
\hline \hline
\end{tabular}


TABLE III. (Upper) Water dipoles, in a.u., for ab initio calculations with different basis sets/basis set extrapolations for a range of molecular configurations. Energies $(E)$ are given relative to the equilibrium geometry, point 1 . The average $C P U$ time required to calculate a single point is also provided. (Lower) Average weighted calculated to measured intensity ratios for selected vibrational bands. The experimental data are due to Birk et al..$^{25,40,43}$ and Loos et al. ${ }^{41}$

\begin{tabular}{|c|c|c|c|c|c|c|c|c|c|}
\hline No. & $\mathrm{R}_{1}$ & $\mathrm{R}_{2}$ & $\theta$ & $\mathrm{E}\left(\mathrm{cm}^{-1}\right)$ & QZ & $5 Z$ & $\mathrm{CBS}^{\mathrm{Q} 5}$ & $6 Z$ & $\mathrm{CBS}^{56}$ \\
\hline 1 & 1.8141 & 1.8141 & 104.52 & 0 & 0.7267 & 0.7282 & 0.7291 & 0.7287 & 0.7290 \\
\hline 2 & 1.68 & 1.98 & 74.00 & 6190.43 & 0.8870 & 0.8890 & 0.8902 & 0.8899 & 0.8904 \\
\hline 3 & 1.94 & 1.94 & 59.00 & 13166.24 & 0.9432 & 0.9454 & 0.9467 & 0.9462 & 0.9467 \\
\hline 4 & 2.10 & 2.18 & 166.00 & 22472.55 & 0.1793 & 0.1798 & 0.1801 & 0.1800 & 0.1801 \\
\hline 5 & 2.10 & 3.00 & 105.00 & 28438.03 & 0.6864 & 0.6893 & 0.6910 & 0.6904 & 0.6910 \\
\hline 6 & 1.60 & 3.50 & 95.00 & 37444.38 & 0.6768 & 0.6792 & 0.6806 & 0.6802 & 0.6808 \\
\hline 7 & 2.00 & 4.00 & 80.00 & 39816.63 & 0.6690 & 0.6691 & 0.6704 & 0.6701 & 0.6707 \\
\hline 8 & 2.80 & 1.85 & 30.00 & 41035.46 & 0.9900 & 0.9924 & 0.9938 & 0.9934 & 0.9940 \\
\hline \multicolumn{5}{|c|}{ CPU TIME (s) } & 15190 & 27684 & 42874 & 84715 & 112399 \\
\hline \multicolumn{10}{|c|}{$\left(v_{1}, v_{2}, v_{3}\right)^{\prime}-\left(v_{1}, v_{2}, v_{3}\right)^{\prime \prime}$} \\
\hline \multicolumn{5}{|c|}{$(100)-(000)$} & 8.42 & 4.74 & 1.99 & 2.83 & 1.48 \\
\hline \multicolumn{5}{|c|}{ (010)-(000) } & 0.67 & 0.95 & 1.16 & 1.11 & 1.23 \\
\hline \multicolumn{5}{|c|}{$(001)-(000)$} & 2.44 & 1.53 & 0.98 & 0.89 & 0.52 \\
\hline \multicolumn{5}{|c|}{$(020)-(000)$} & 2.17 & 1.32 & 0.70 & 0.73 & 0.44 \\
\hline \multicolumn{5}{|c|}{$(121)-(000)$} & 1.25 & 1.71 & 1.78 & 0.80 & 0.62 \\
\hline
\end{tabular}

require less than $50 \%$ of the $\mathrm{CPU}$ time to compute than the augcc-CV6Z calculations. The marginal difference between the $\mathrm{CBS}^{\mathrm{Q} 5}$ and $\mathrm{CBS}^{56}$ dipoles highlights the correlation between these basis sets. This marginal deviation also holds true for those dipoles computed for geometries with a large proportion of stretch and/or bend (see Table III), which means that the technique holds true for all regions of the DMS. This result implies that highly accurate, global dipole surfaces can now be calculated with lower levels of theory at a fraction of the CPU cost, with marginal loss of precision. This should prove to be important for molecules that are too large to compute large grids of energies with an aug-cc-pCV6Z basis set.
In Table III, the $\mathrm{CBS}^{56}$ dipoles are consistently larger than those calculated with the aug-cc-pCV6Z basis set and should be of a comparable magnitude to those calculations expected with a aug-cc-pCV7Z basis set, if the smooth exponential growth of the dipole remains consistent. Obtaining these $\mathrm{CBS}^{56}$ dipoles will be computationally expensive for all molecules, as calculations will be required at both the aug-cc-pCV5Z and aug-cc-pCV6Z levels of theory. This will be limited to the lighter molecules.

Birk et al. ${ }^{25}$ analyzed transition intensities of $\mathrm{H}_{2} \mathrm{O}$ in the $\mathrm{IR}^{40,41,43}$ and found the most recent (at the time) ab initio line list to deviate from the experimental observations ${ }^{41}$ in the $v_{1}$ fundamental

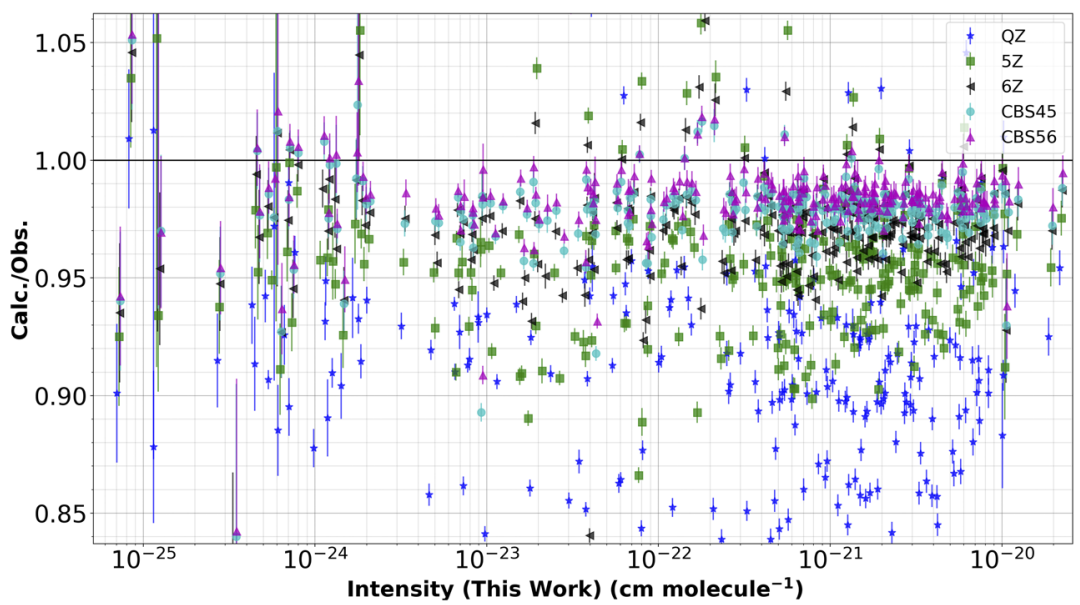

FIG. 1. Ratios of calculated $v_{1}$ transition intensities obtained from each dipole moment surface against experimental measurements of Birk et al. ${ }^{40}$ and Loos et al. ${ }^{41}$ The error bars are experimental. 


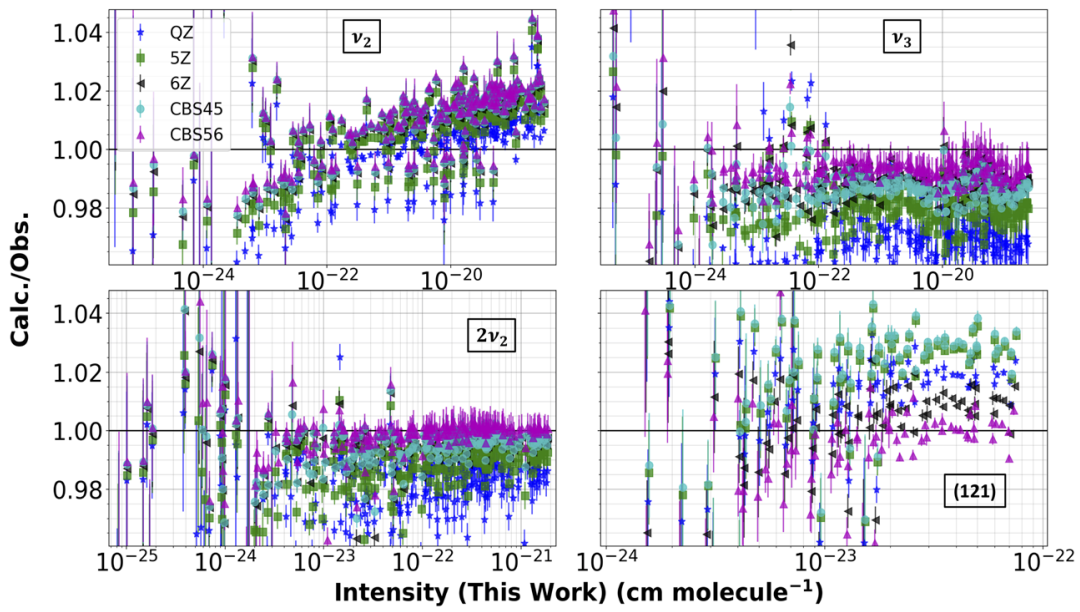

FIG. 2. Ratios of intensities calculated using DMS from Table | to the experimental measurements of Birk et al. ${ }^{25,40,43}$ and Loos et al. ${ }^{41}$ shown for $v_{3},{ }^{40,41} v_{2},{ }^{43} 2 v_{2},{ }^{41}$ and $(121) .{ }^{25}$ by $3 \%-15 \%$. This ab initio line list was calculated with the LTP2011S DMS of Lodi et al. ${ }^{30}$ Despite this large residual, all other bands in the $3000-4400 \mathrm{~cm}^{-1}$ region showed good agreement with experiment, suggesting the presence of an issue in the theoretical model. We recently calculated a new line list with the CKAPTEN DMS, and there were a large number of improvements over the recent POKAZATEL line list, ${ }^{17}$ particularly for those transitions with wavelengths below $1 \mu \mathrm{m}\left(\omega>10000 \mathrm{~cm}^{-1}\right)$. The same conclusion was made for the isotopologs. The POKAZATEL line list was computed with a variation of the LTP2011 DMS dipole surface from Lodi et al., termed LTP2011S, which utilized fewer parameters for improved stability in highly energetic regions. However, our calculated intensities in the $v_{1}$ fundamental showed no signs of improvement over POKAZATEL nor what was computed in 2011. Over 16000 aug-cc-pCV6Z dipoles underlay this CKAPTEN surface; hence, discrepancies in this band are not due to the number of points fit nor is it a fitting issue as different functional forms were used for each of these models. These line lists used rotation-vibration wavefunctions that were also calculated with a variety of potential energy surfaces; thus, these are also not the source of the deviation, although any energy dependence in the residuals will be due to the potential.

Figure 1 plots the ratios of our calculated transition intensities in $v_{1}$ to those measurements from Loos et al. ${ }^{41}$ As the level of theory increases from QZ through to $\mathrm{CBS}^{56}$, the deviation in the $v_{1}$ fundamental reduces from $2.83 \%$ with the $6 \mathrm{Z}$ DMS to only $1.48 \%$ with the new $\mathrm{CBS}^{56}$ DMS (see Table III). Indeed, the spectral intensities calculated with the $\mathrm{CBS}^{\mathrm{Q} 5}$ DMS are closer to the experimental values of Loos et al. ${ }^{41}$ than the aug-cc-pCV6Z calculations. This suggests that better results can potentially be obtained by extrapolating the computationally less expensive calculations. To verify that the extrapolation technique works on a global scale, we need to also investigate other infrared bands measured by Loos et al. and Birk et al. ${ }^{40,43}$ Ratios of transition intensities in $v_{3}, v_{2}$, and $2 v_{2}$ are presented in Fig. 2. In addition, Table III presents a general overview of several other bands, which we do not present in our figures.

For the $v_{3}$ and $2 v_{2}$ bands, shown in Fig. 2 , the computed $\mathrm{CBS}^{56}$ spectrum is again closer to the experimental intensities than any of

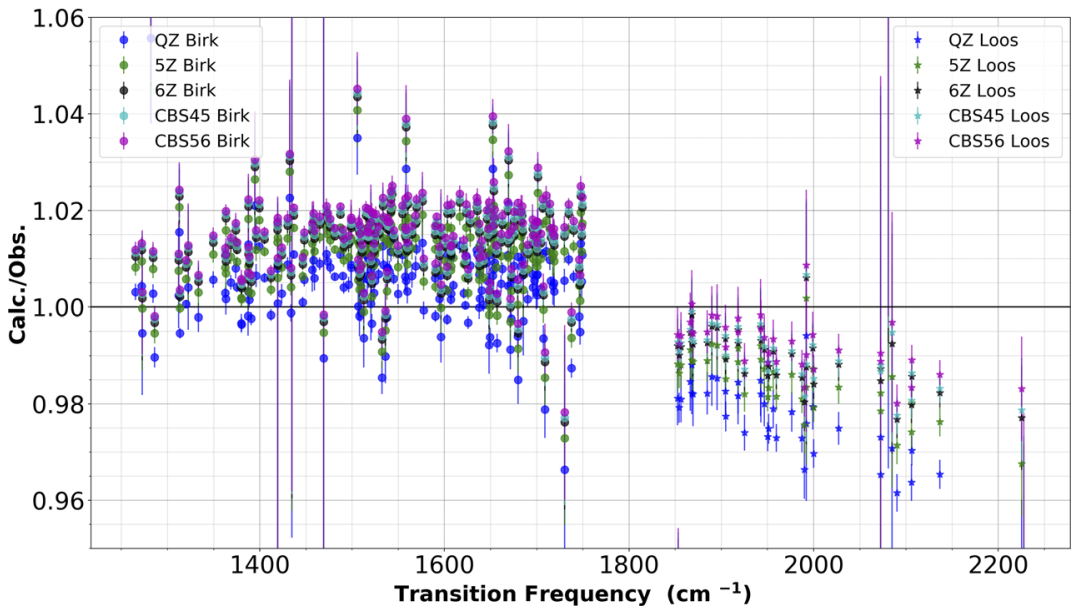

FIG. 3. Ratios of intensities calculated using the DMS from Table I to the experimental measurements of Birk et al..$^{40}$ and Loos et al..$^{41}$ for $v_{2}$ transitions plotted as a function of frequency. 
the other datasets. However, the $v_{2}$ ratios suggest that the analysis of the experimental spectrum of Loos et al. ${ }^{41}$ differs from that of Birk et al. ${ }^{40}$ In Fig. 3, we plot intensity ratios for $v_{2}$ transitions as a function of frequency and separate the datasets. Birk analyzed the spectrum with a speed-dependent Voigt profile, while Loos used a quadratic speed dependent hard collision profile with Rosenkranz line-mixing. Our results show that the choice of profile is very important for high-accuracy intensity measurements.

For more energetic transitions, wavelengths shorter than $1 \mu \mathrm{m}$, the $\mathrm{CBS}^{56}$ intensities show excellent agreement with the measurements of Birk et al. ${ }^{25}$ (see Fig. 2). For the same band, the calculated aug-cc-pCVQZ spectrum is closer to the experimental data than the $\mathrm{CBS}^{\mathrm{Q} 5}$ and aug-cc-pCV5Z spectra, which is counterintuitive. We also expect the $\mathrm{CBS}^{\mathrm{Q} 5}$ spectrum to be closer to experiment than the aug-cc-pCV5Z spectrum, which is also not the case. The results indicate there may be an underlying issue regarding the aug-cc-pCVQZ dataset in this band.

\section{CONCLUSION}

We compute $a b$ initio dipoles with the MRCI procedure embedded within the quantum chemistry package $\mathrm{MOLPRO}^{36}$ with the aug-cc-pCV $(\mathrm{X}=\mathrm{Q}, 5,6) \mathrm{Z}$ basis sets ${ }^{26-28,36}$ for water vapor. Using these results, we similarly created extrapolated $\mathrm{CBS}^{\mathrm{Q} 5}$ and $\mathrm{CBS}^{56}$ dipoles, each obtained by extrapolating energies from the augcc-pCVQZ and aug-cc-pCV5Z calculations and likewise from the aug-cc-pCV5Z and aug-cc-pCV6Z levels of theory. The same functional form was fit to these dipole sets to create five individual dipole moment surfaces.

For a select number of global geometries studied, the extrapolated $\mathrm{CBS}^{\mathrm{Q} 5}$ and $\mathrm{CBS}^{56}$ dipoles are within $10^{-4}$ a.u. of each other. This finding does reflect the correlation in the aug-cc-pCV $(X=Q, 5,6)$ basis sets. The greatest achievement surrounds the computational time required for calculating the $\mathrm{CBS}^{\mathrm{Q} 5}$ dipoles: they require 50\% less CPU time compared to the aug-cc-pCV6Z dipoles.

Using the potential energy surface of Mizus et al., known as PES15K, which predicts energy levels below $15000 \mathrm{~cm}^{-1}$ to a root mean square of $0.011 \mathrm{~cm}^{-1}$, we create wavefunctions up to total quantum number $J=6$. With these, we calculate spectra for each of the dipole surfaces with an upper threshold of $15000 \mathrm{~cm}^{-1}$ and where possible, we replace calculated energy levels with those in the MARVEL database.

The transition intensities obtained from each dataset are compared against the experimental measurements of Birk et al. and Loos et al., which are both in the IR. Overall, the $\mathrm{CBS}^{56}$ DMS, despite being fit with dipoles that are only accurate to the fourth significant figure, resulting intensities exhibit the best agreement with the measurements, and for several bands, the deviation is in the subone-percent range. Also, comparing the $\mathrm{CBS}^{56}$ and aug-cc-pCV6Z results indicates that it may be preferable, in cases, to consider using the $\mathrm{CBS}^{56}$ dipoles over the aug-cc-pCV6Z dipoles.

For those strong transitions in the IR, calculated intensities obtained using the $\mathrm{CBS}^{\mathrm{Q} 5}$ and $\mathrm{CBS}^{56}$ DMS's suggest that the limit of dipole precision required for obtaining subpercent accuracy against high-quality experiments could be on the order of $10^{-4}$ a.u.

We show that the two point formula works very well for extrapolating dipoles, reducing the deviation from experimental measurements by approximately one percent in certain bands, and thus, we estimate that the application of a three-point formula for dipole extrapolation could potentially improve the accuracy by another $0.5 \%$.

Coupled-cluster methods are, in general, computationally less expensive than MRCI but, nonetheless, provide accurate results for energies and hence finite field dipoles for geometries close to equilibrium. ${ }^{45-47}$ The extrapolation technique is successful not because of the underlying method of calculation, in this case MRCI, but because of the nature of the aug-cc-pCV(X $=\mathrm{Q}, 5,6) \mathrm{Z}$ basis sets. We, therefore, expect the extrapolation technique to work for coupled-cluster calculations, providing the calculations remain converged. Applying this extrapolation technique to high-level coupled-cluster energies may prove to be a cheaper alternative of obtaining mid-/lower-level MRCI results.

\section{ACKNOWLEDGMENTS}

This work was supported by the UK's Natural Environment Research Council (NERC) under Grant No. NE/T000767/1. The authors acknowledge the use of the UCL Grace, Legion and Myriad High Performance and High Throughput Computing Facilities (Grace@UCL, Legion@UCL, Myriad@UCL), and associated support services, in the completion of this work.

\section{REFERENCES}

${ }^{1}$ P. Zoogman, X. Liu, R. M. Suleiman, W. F. Pennington, D. E. Flittner, J. A. Al-Saadi, B. B. Hilton, D. K. Nicks, M. J. Newchurch, J. L. Carr et al., J. Quant. Spectrosc. Radiat. Transfer 186, 17 (2017).

${ }^{2}$ K. Weigel, A. Rozanov, F. Azam, K. Bramstedt, R. Damadeo, K.-U. Eichmann, C. Gebhardt, D. Hurst, M. Kraemer, S. Lossow et al., Atmos. Meas. Tech. 9, 133 (2016).

${ }^{3}$ P. F. Levelt, J. Joiner, J. Tamminen, J. P. Veefkind, P. K. Bhartia, D. C. Stein Zweers, B. N. Duncan, D. G. Streets, H. Eskes, R. van der A et al., Atmos. Chem. Phys. 18, 5699 (2018).

${ }^{4}$ S. T. Gulde, M. G. Kolm, D. J. Smith, R. Maurer, G. B. Courrèges-Lacoste, M. Sallusti, and G. Bagnasco, Proc. SPIE 10563, 1056341 (2017).

${ }^{5}$ S. Noël, M. Buchwitz, H. Bovensmann, R. Hoogen, and J. P. Burrows, Geophys. Res. Lett. 26, 1841, https://doi.org/10.1029/1999gl900437 (1999).

${ }^{6}$ A. Kuze, H. Suto, M. Nakajima, and T. Hamazaki, Appl. Opt. 48, 6716 (2009).

${ }^{7}$ D. Crisp and C. Johnson, Acta Astronaut. 56, 193 (2005).

${ }^{8}$ I. E. Gordon et al., J. Quant. Spectrosc. Radiat. Transfer 203, 3 (2017).

${ }^{9}$ S. Vasilchenko, H. Tran, D. Mondelain, S. Kassi, and A. Campargue, J. Quant. Spectrosc. Radiat. Transfer 235, 332 (2019).

${ }^{10}$ S. Mikhailenko, D. Mondelain, E. Karlovets, S. Kassi, and A. Campargue, J. Quant. Spectrosc. Radiat. Transfer 206, 163 (2018).

${ }^{11}$ D. Mondelain, S. Mikhailenko, E. Karlovets, S. Bacguier, S. Kassi, and A. Campargue, J. Quant. Spectrosc. Radiat. Transfer 203, 206 (2017).

${ }^{12}$ X. Huang, D. W. Schwenke, S. A. Tashkun, and T. J. Lee, J. Chem. Phys. 136, 124311 (2012).

${ }^{13}$ I. I. Bubukina, O. L. Polyansky, N. F. Zobov, and S. N. Yurchenko, Opt. Spectrosc. 110, 160 (2011).

${ }^{14}$ I. I. Mizus, A. A. Kyuberis, N. F. Zobov, V. Y. Makhnev, O. L. Polyansky, and J. Tennyson, Philos. Trans. R. Soc., A 376, 20170149 (2018).

${ }^{15}$ S. N. Yurchenko, M. Carvajal, P. Jensen, F. Herregodts, and T. R. Huet, Chem. Phys. 290, 59 (2003).

${ }^{16}$ M. J. Down, J. Tennyson, M. Hara, Y. Hatano, and K. Kobayashi, J. Mol. Spectrosc. 289, 35 (2013)

${ }^{17}$ O. L. Polyansky, A. A. Kyuberis, N. F. Zobov, J. Tennyson, S. N. Yurchenko, and L. Lodi, Mon. Not. R. Astron. Soc. 480, 2597 (2018).

${ }^{18}$ D. W. Schwenke, J. Phys. Chem. A 105, 2352 (2001). 
${ }^{19}$ O. L. Polyansky, A. G. Császár, S. V. Shirin, N. F. Zobov, P. Barletta, J. Tennyson, D. W. Schwenke, and P. J. Knowles, Science 299, 539 (2003).

${ }^{20}$ H. M. Quiney, P. Barletta, G. Tarczay, A. G. Császár, O. L. Polyansky, and J. Tennyson, Chem. Phys. Lett. 344, 413 (2001).

${ }^{21}$ O. L. Polyansky, R. I. Ovsyannikov, A. A. Kyuberis, L. Lodi, J. Tennyson, and N. F. Zobov, J. Phys. Chem. A 117, 9633-9643 (2013).

${ }^{22}$ S. Mikhailenko, E. Karlovets, S. Vasilchenko, D. Mondelain, S. Kassi, and A. Campargue, J. Quant. Spectrosc. Radiat. Transfer 236, 106574 (2019).

${ }^{23}$ O. L. Polyansky, K. Bielska, M. Ghysels, L. Lodi, N. F. Zobov, J. T. Hodges, and J. Tennyson, Phys. Rev. Lett. 114, 243001 (2015).

${ }^{24}$ E. J. Zak, J. Tennyson, O. L. Polyansky, L. Lodi, S. A. Tashkun, and V. I. Perevalov, J. Quant. Spectrosc. Radiat. Transfer 177, 31 (2016).

${ }^{25}$ M. Birk, G. Wagner, J. Loos, L. Lodi, O. L. Polyansky, A. A. Kyuberis, N. F. Zobov, and J. Tennyson, J. Quant. Spectrosc. Radiat. Transfer 203, 88 (2017).

${ }^{26}$ T. H. Dunning, J. Chem. Phys. 90, 1007 (1989).

${ }^{27}$ D. E. Woon and T. H. Dunning, Jr., J. Chem. Phys. 103, 4572 (1995).

${ }^{28}$ K. A. Peterson and T. H. Dunning, J. Chem. Phys. 117, 10548 (2002).

${ }^{29}$ E. K. Conway, A. A. Kyuberis, O. L. Polyansky, J. Tennyson, and N. Zobov, J. Chem. Phys. 149, 084307 (2018).

${ }^{30}$ L. Lodi, J. Tennyson, and O. L. Polyansky, J. Chem. Phys. 135, 034113 (2011).

${ }^{31}$ H. Partridge and D. W. Schwenke, J. Chem. Phys. 106, 4618 (1997).

${ }^{32}$ D. W. Schwenke and H. Partridge, J. Chem. Phys. 113, 6592 (2000).

${ }^{33}$ X. Huang, D. W. Schwenke, and T. J. Lee, J. Quant. Spectrosc. Radiat. Transfer 230, 222 (2019).

${ }^{34}$ O. L. Polyansky, N. F. Zobov, I. I. Mizus, A. A. Kyuberis, L. Lodi, and J. Tennyson, J. Quant. Spectrosc. Radiat. Transfer 210, 127 (2018).
${ }^{35}$ V. G. Tyuterev, A. Barbe, D. Jacquemart, C. Janssen, S. N. Mikhailenko, and E. N. Starikova, J. Chem. Phys. 150, 184303 (2019).

${ }^{36}$ H.-J. Werner, P. J. Knowles, G. Knizia, F. R. Manby, and M. Schütz, Wiley Interdiscip. Rev.: Comput. Mol. Sci. 2, 242 (2012).

${ }^{37}$ E. K. Conway, I. E. Gordon, A. A. Kyuberis, O. L. Polyansky, J. Tennyson, and N. F. Zobov, J. Quant. Spectrosc. Radiat. Transfer 241, 106711 (2019).

${ }^{38}$ D. Feller, J. Chem. Phys. 98, 7059 (1993).

${ }^{39}$ P. Barletta, S. V. Shirin, N. F. Zobov, O. L. Polyansky, J. Tennyson, E. F. Valeev, and A. G. Császár, J. Chem. Phys. 125, 204307 (2006).

${ }^{40}$ M. Birk, J. Loos, G. Wagner, D. Mondelain, S. Kassi, A. Campargue, F. Hase, J. Orphal, A. Perrin, L. Coudert et al., in Proceedings of the ESA Living Planet Symposium, Prague, Czech Republic, 2016.

${ }^{41}$ J. Loos, M. Birk, and G. Wagner, J. Quant. Spectrosc. Radiat. Transfer 203, 103 (2017).

${ }^{42}$ J. Tennyson, M. A. Kostin, P. Barletta, G. J. Harris, O. L. Polyansky, J. Ramanlal, and N. F. Zobov, Comput. Phys. Commun. 163, 85 (2004).

${ }^{43}$ M. Birk and G. Wagner, J. Quant. Spectrosc. Radiat. Transfer 170, 159 (2016).

${ }^{44}$ T. Helgaker, W. Klopper, H. Koch, and J. Noga, J. Chem. Phys. 106, 9639 (1997).

${ }^{45}$ K. L. Chubb, A. Yachmenev, J. Tennyson, and S. N. Yurchenko, J. Chem. Phys. 149, 014101 (2018).

${ }^{46}$ P. A. Coles, S. N. Yurchenko, R. P. Kovacich, J. Hobby, and J. Tennyson, Phys. Chem. Chem. Phys. 21, 3264 (2019).

${ }^{47}$ A. Owens, A. Yachmenev, W. Thiel, A. Fateev, J. Tennyson, and S. N. Yurchenko, Mon. Not. R. Astron. Soc. 479, 3002 (2018). 